\title{
Los planes de negocios y los proyectos de inversión: similitudes y diferencias
}

\section{RESUMEN}

El presente artículo brinda información sobre los términos frecuentemente utilizados en el inicio y mejora de actividades empresariales, ellos son: los planes de negocio y proyectos de inversión; con tal propósito se describen las similitudes y diferencias, haciendo uso de los conceptos, objetivos y estructuras definidas en la bibliografia de la especialidad.

Palabras clave: proyecto de inversión, planes de negocio, idea de negocio, emprendedor

LOS PLANES DE NEGOCIOS Y LOS PROYECTOS DE INVERSIÓN: SIMILITUDES Y DIFERENCIAS

\section{ABSTRACT}

This article aims to provide information about the terms frequently used in the home and improvement of business activities, they are: the of business plans and investment projects; for such purpose describes the similarities and differences, making use of the concepts, objectives and structures defined in the literature of speciality.

Keywords: investment project, business plan, business idea, entrepreneur

\section{INTRODUCCIÓN}

El auge de la economía en el Perú ha dinamizado los mercados generando mayores ingresos en la población, origina mayor interés en cubrir sus necesidades insatisfechas, ello lleva a oportunidades de negocio, por lo tanto, el riesgo de fracasar en la realización de un negocio sin un documento o guía es elevado. Es por ello, los términos de proyectos de inversión y planes de negocio son utilizados con mayor frecuencia ya sea por el sector público y privado.

\section{PLAN DE NEGOCIO}

Un plan de negocios "es un documento escrito de manera sencilla y precisa, el cual es el resultado de una planificación. Este documento muestra los objetivos que se quieren obtener y las actividades que se desarrollarán para lograr dichos objetivos" [12].

De igual manera, se puede explicar "un plan de negocio como un instrumento de gestión de la empresa que sirve de guía para el emprendedor o empresario implemente un negocio. Es decir, el plan de negocio, es un instrumento de planificación que permite comunicar una idea de negocio para gestionar su financiamiento" [2].

Todo plan "tiene etapas predecibles que involucran una serie de tareas delimitadas en un tiempo, es decir, comienzan con el estudio de una idea y terminan con la entrega de un plan de negocio concluido" [2].

"Un plan de negocios es útil para ayudar a conocer el negocio en detalle, es decir, sus antecedentes, las estrategias, factores de éxito o fracaso y las metas" [9]

La bibliografía sobre los planes de negocio incide en las características y habilidades de la persona que inicia el negocio. Es así que en el Manual de Jóvenes Emprendedores, elaborado por el Ministerio de Trabajo se menciona: "Las personas emprendedoras tienden a canalizar su potencialidad hacia la generación de negocios. Saben que para ser empresario se necesita ganas de hacer bien las cosas, tener ideas innovadoras y utilizar adecuadamente cada una de sus habilidades a fin

\footnotetext{
Ingeniero Industrial, Profesor en la Facultad de Ingeniería Industrial. E-mail: cieandia@hotmail.com

** Estudiante de la Facultad de Ingeniería Industrial.
} 
de responder a las necesidades de un mercado determinado" [9].

Así también, el Centro de Emprendimiento Universidad San Ignacio de Loyola en su manual de emprendedores, señala "un emprendedor es quien, impulsado por un sueño, una idea de negocio, el deseo de cambiar su entorno o la necesidad de materializar un plan de vida o de negocios, asume el reto de concretarlo desafiando paradigmas, enfrentando escenarios cambiantes y superando dificultades que suelen ser difíciles para otras personas. Estas ideas emanan de su mente, es decir, son capaces de identificar oportunidades de negocio donde otros no pueden ver." [4]

Prolnversión enfatiza que "El plan de negocio es una herramienta clave para las pequeñas empresas" [8].

En la Figura 1 se define la estructura de un plan de negocios establecida por Weinberger:

\section{ESTRUCTURA DE UN PLAN DE NEGOCIO}

Según la guía para el desarrollo de la micro y pequeña empresa [9], un plan de negocio contiene la siguiente estructura:

1. Resumen ejecutivo: resumen y conclusiones.

2. Descripción y visión del negocio: información que permita entender el negocio y la descripción de los productos y los servicios que ofrece.

3. Análisis del mercado: descripción del sector en el que el negocio compite o competirá.

4. Planeamiento estratégico: una estrategia de planeamiento que nos permita saber dónde estamos y hacia dónde vamos, así como cuáles son nuestras fortalezas y debilidades.

5. Estrategia de comercialización y ventas: estrategia que se va a seguir para distribuir y vender los productos.

6. Análisis del proceso productivo: cómo se tiene que organizar para producir, qué insumos, maquinaria, etc., son necesarios.

7. Análisis económico financiero: cuántos ingresos y egresos se va a tener.

\section{PROYECTOS DE INVERSIÓN}

Desde una perspectiva general, "proyecto de inversión se entiende como una intervención en un determinado medio para dar solución a una problemática existente y conseguir el objetivo deseado.
Dicho problema se puede percibir como una limitación o un exceso de un bien y/o servicio" [1].

Un proyecto "es una serie de planteamientos encaminados a la producción de un bien o la prestación de servicios, con empleo de una cierta metodología y con miras a obtener determinados resultados" [7].

Los proyectos de inversión por su naturaleza están ligados a propuestas de sistemas complejos de negocios como es el caso de plantas industriales, pero ello no excluye a que pueda ser aplicado a negocios pequeños.

\section{ESTRUCTURA DE UN PROYECTO DE INVER- SIÓN}

Dentro de la estructura de un proyecto de inversión se encuentran un conjunto de estudios, los cuales permitirán sintetizar la información del negocio. Estos estudios se muestran en la Figura N. ${ }^{\circ} 2$.

1. Estudio legal: determinar la existencia de restricciones en la realización del proyecto (viabilidad legal), relacionada a la normatividad.

2. Estudio de mercado: permite cuantificar la población al cual se les puede ofrecer el producto y/o servicio. Además, se establecen las estrategias de ingreso al mercado.

3. Estudio técnico: permite describir elementos técnicos (tamaño, localización, proceso productivo, etc.).

4. Estudio organización: determina la organización administrativa que tendrá el negocio.

5. Estudio ambiental: determina los efectos del proyecto sobre el medio ambiente y viceversa.

6. Estudio económico-financiero: determina la viabilidad del proyecto a partir de indicadores de rentabilidad.

\section{SIMILITUDES Y DIFERENCIAS}

Los proyectos de inversión se inician bajo un enfoque de generación de empresas productivas, por lo que su lenguaje e instrumentos fueron muy técnicos inicialmente; en la actualidad dicho enfoque se está adecuando a las nuevas tendencias de los negocios, ello implica incorporar a un nuevo segmento de análisis: la micro y pequeña empresa, estrategias de ingreso al mercado, perspectivas ambientales, etc. 
Figura 1. Estructura de un plan de negocios.

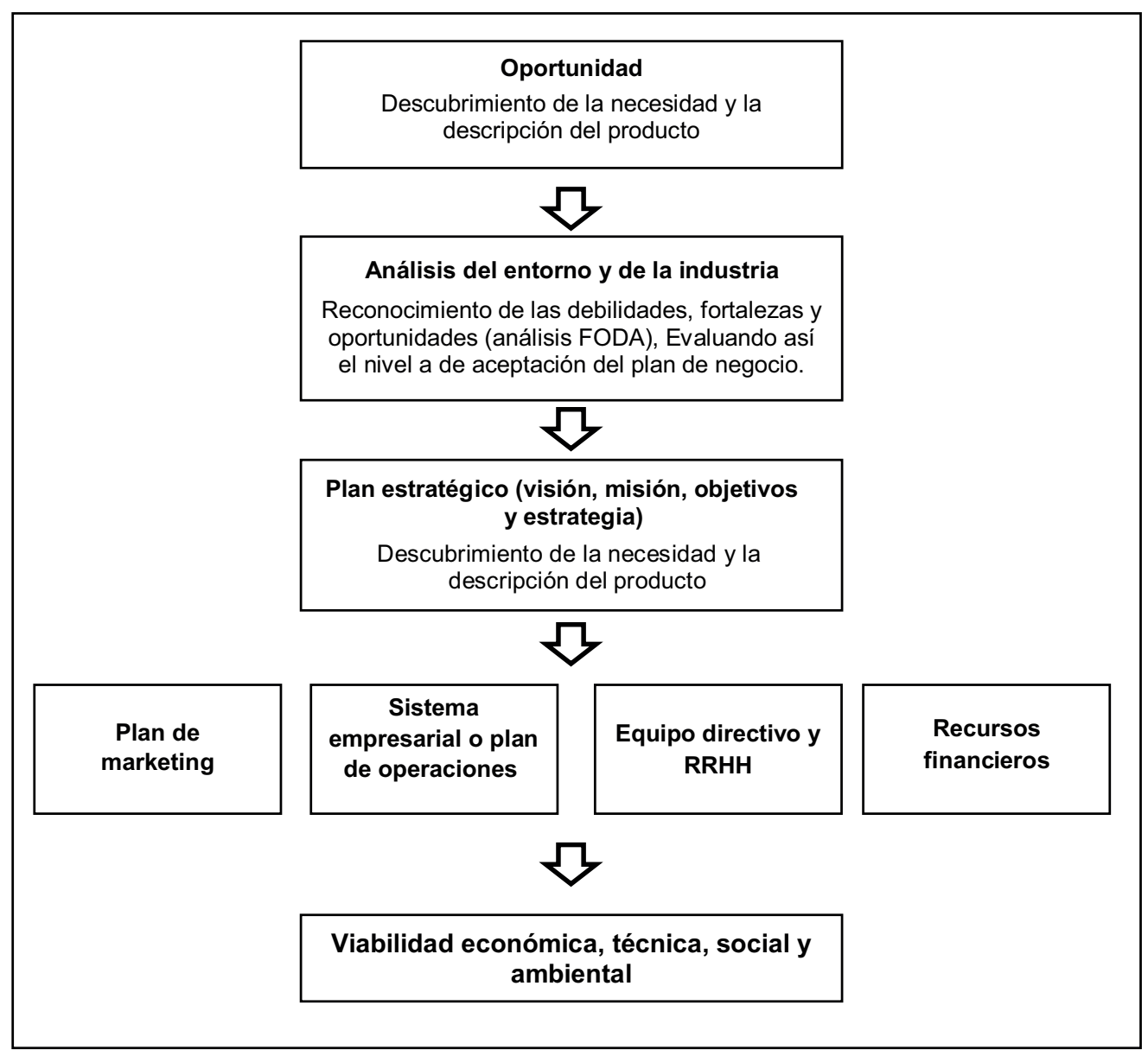

Fuente: Tomado de la referencia [12].

Figura 2. Estructura de un proyecto de inversión.

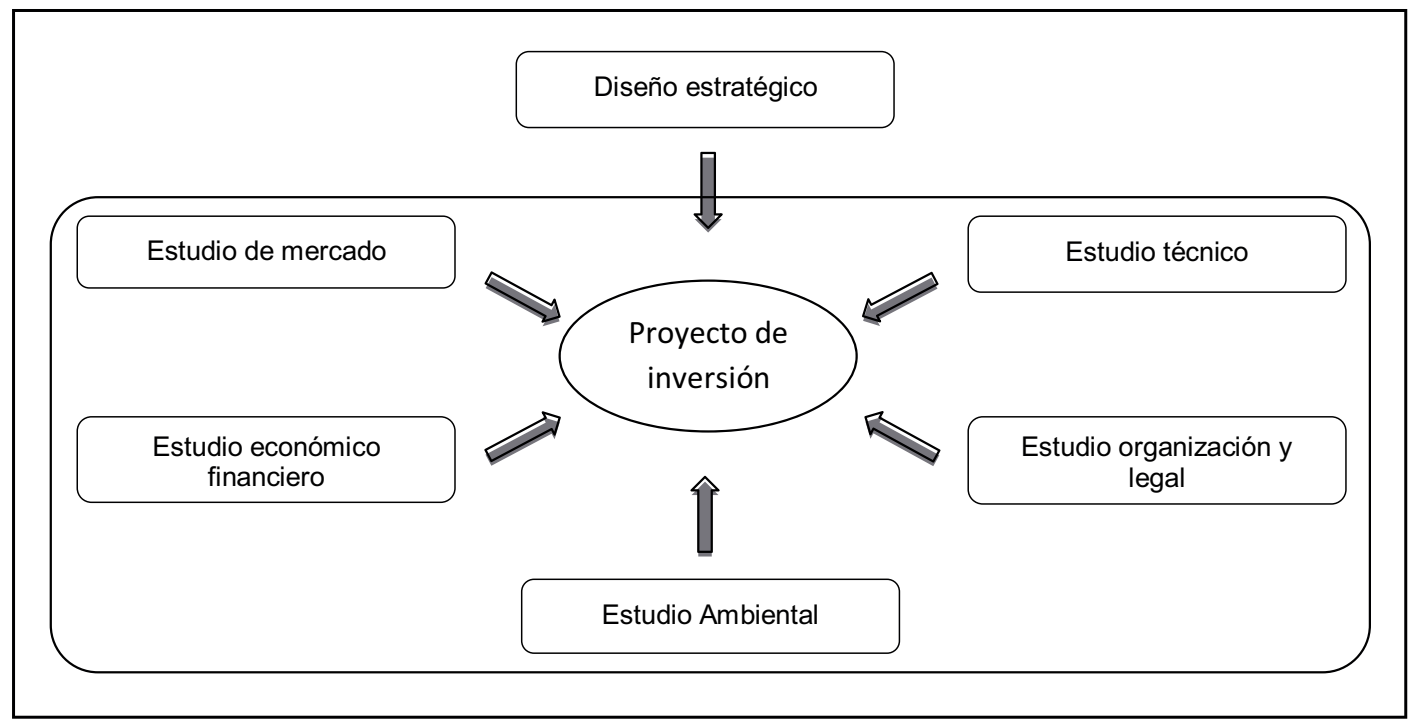

Fuente: Tomado de la referencia [1]. 
Figura 3. Similitudes entre planes de negocio y proyectos de inversión.

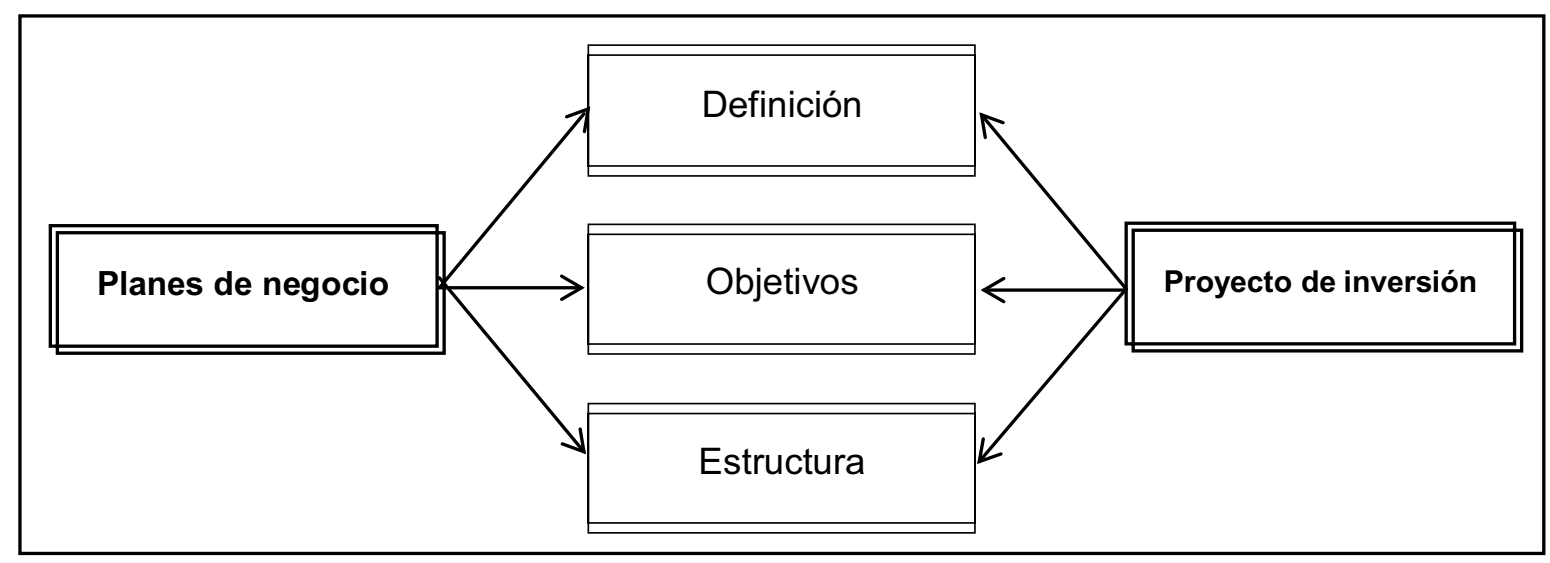

Fuente: Elaboración propia.

Los planes de negocio surgen como una propuesta de resolver la necesidad de emprender un negocio relacionado generalmente a comercialización de algún tipo de servicio en forma personal.

Por lo tanto, ambas tienen el mismo enfoque, siendo la única diferencia su aplicación. Los proyectos de inversión usualmente tienen un uso organizacional, mientras que los planes de negocio tienen una tendencia unipersonal de negocios. Ambos términos tienen un fin común, ser un documento que sintetice lo que se desea lograr en la implementación de una actividad empresarial ya sea personal y/o organizacional. Ello se puede verificar en el siguiente enunciado: "un proyecto de inversión viene a ser una idea de negocio que tiene un emprendedor, se determina su viabilidad a través de la evaluación de sus aspectos comerciales, técnicos, de gestión, legal, económicos, financieros y ambientales" [4].

Las similitudes encontradas en ambos términos se presentan en su definición, objetivos estructura y estudios.

\section{CONCLUSIONES}

- Un plan de negocios al igual que un proyecto de inversión surge debido a la existencia de oportunidades de negocios que pueden ser necesidades no satisfechas de los usuarios.

- Ambos conceptos tienen el mismo enfoque, ser un documento que sintetice lo que se desea lograr en la implementación de una actividad empresarial, ya sea personal y/o organizacional.
- La única diferencia en ambas terminologías es su aplicación, los proyectos de inversión usualmente tienen una aplicación organizacional, mientras que los planes de negocio tienen una tendencia unipersonal de negocios.

- Ambos términos generan oportunidades de mejora del servicio, dinamismo y actualización del negocio.

\section{BIBLIOGRAFÍA}

[1] Andía Valencia Walter (2012). Proyectos de Inversión. 3. ${ }^{a}$ ed. El Saber. Perú.

[2] Centro de Apoyo al Sector Empresarial CEASE (2011). Elaboración de Plan de Negocio para MYPE. http://www.fondoitaloperuano.org/wpcontent/uploads/2012/01/Elaboraci\%C3\%B3nplan-de-negocio-para-MYPE.pdf (visitado el 02-08-2013).

[3] Centro de emprendimiento Universidad San Ignacio de Loyola. Manual de Emprendedores. http://ceusil.usil.edu.pe/manual-deemprendedores/ (visitado el 03-07-2013).

[4] Flores Uribe Juan A. (2012). Plan de Negocios para pequeñas empresas. 1. ${ }^{\mathrm{a}}$ ed. U-Transversal. Colombia.

[5] Flórez Uribe Juan A. (2007). Proyecto de inversión para las PYME. 1. ${ }^{a}$ ed. Eco Editores. Colombia.

[6] Hernández Hernández Abraham y otros (2005). Formulación y Elaboración de Proyectos 
de Inversión. 5. ${ }^{a}$ ed. International Thomson Editores, México.

[8] Programa Conjunto Juventud. Empleo y Migración (2009). Manual Jóvenes Emprendedores. http://conjoven.oit.org. pe/wp-content/uploads/2010/10/ManualJ\%C3\%B3venes-Emprendedores-GeneranIdeas-de-Negocios.pdf (visitado el 22-07-2013).

[9] Prolnversión (2007). Guía para el desarrollo de la micro y pequeña empresa. http://www. proinversion.gob.pe/RepositorioAPS/0/0/
arc/GUIAS/MYPEquenaEmpresaCrece.pdf (visitado el 09-07-2013).

[10] Thomsen Mogens. El Plan de Negocios Dinámico. 1. ${ }^{a}$ ed. Copyright Thomsen Business Information.

[11] Viniegra Sergio (2007). Entendiendo el Plan de Negocios. 1. ${ }^{a}$ ed. Copyright.

[12] Weinberger Villarán Karen (2009). Plan de Negocios. http://www.crecemype.pe/portal/ images/stories/files/plan_negocios.pdf (visitado el 08-07-2013). 